\title{
An algorithm for the unambiguous determination of the equidistant boundary line between two (or more) coastlines
}

\author{
Stefano Ferrero • Marco Pierozzi • Luca Repetti • \\ Luciano Surace
}

Received: 23 October 2008 / Accepted: 14 July 2009 /Published online: 5 August 2009

(C) Società Italiana di Fotogrammetria e Topografia (SIFET) 2009

\begin{abstract}
This paper deals with the problem of the rigorous determination of a marine boundary line between two or more countries, both from the theoretical and the algorithmic point of view. Through suitable simplifications, also the problem of the determination of the territorial limit is discussed and solved. Both the applications above are surely important from the geomatic and geo-political point of view, also outside the peculiar problems relevant to the marine environment.
\end{abstract}

Keywords Boundary line $\cdot$ Coastline $\cdot$ Equidistant point . Median line

\section{Introduction}

The need for an unambiguous, rigorous, and easily testable definition of equidistant point (EP) which can be exploited for the determination of marine boundary lines and territorial limits requires the development of methods for the determination of the geometric locus of points equidistant from two or more point sets.

Since such point sets as coastlines and baselines ${ }^{1}$ are dealt with, which in general are not easily represented

\footnotetext{
${ }^{1}$ This term generally indicates the line from which the amplitude of the territorial limit is measured. The types of baselines conceived by the international rules depending on the geographic situation of the specific area are: (a) the "normal baseline" coinciding with the lowtide line along the coast; (b) the "straight baseline", connecting suitable points along the coast; (c) the "archipelagic baseline", consisting in a special straight baseline connecting the extreme points of the islands and the external rocks of an archipelagic country.
}

S. Ferrero $\cdot$ M. Pierozzi $(\bowtie) \cdot$ L. Repetti $\cdot$ L. Surace

Istituto Idrografico della Marina,

Genova, Italy

e-mail: pierozzi@tele2.it analytically, the use of numerical calculus appears necessary in order to develop a satisfactory algorithm suitable to the different precision requirements which can be conceived in view of the specific purposes.

On the other hand, the definition of equidistant line or median line (the median line is a line every point of which is equidistant from nearest points on the baselines of two states; IHO, IAG, IOC 2006) explicitly applies the equidistance criteria to the points belonging to the baselines in addition to the median line points. As a consequence, to conceive the coastlines as point sets, and to deal with them accordingly, is necessary and central to the method presented in this work.

\section{The classical method}

The method adopted by the Italian Navy Hydrographic Institute, which is widely adopted internationally as well, makes reference to the method developed by the British Navy Commander R.H. Kennedy and presented at the Geneva Conference of the year 1958 by the British delegation in a paper describing the practical construction of an equidistance line both in the case of opposite coasts and in the case of contiguous ones: that paper represented for the cartographers the only and most valid reference for a long time (Francalanci and Spanio 2000).

The nautical map represents mostly the sea including its coastal boundaries and the relevant hydrographic and morphologic features. At a small scale, one can have a large view which allows to decide if a boundary line divides the space in a more or less fair manner; anyway, a geometric procedure must make reference to well defined and represented fixed points (a condition which often is not fulfilled due to the main features of a cartographic 
document); on the other hand, the large-scale maps usually deal with coastal regions with limited sea areas.

Thus, in the 1958 Convention, two main criteria were discussed in order to solve the boundary problems:

- the agreements between neighboring countries;

- the equidistance line as a limit which no one is allowed to trespass.

As a matter of fact, the equidistance line cannot be the only method which provides the solutions to every problems; indeed, since the first 1958 document the equidistance line is considered "in case of lack of specific agreement, unless another boundary line can be chosen according to special circumstances"; therefore, a suitably determined equidistance line can represent the first approach towards the definition of a median line (often used a synonym) which, in particular cases, can result fair and acceptable to both parties and, in principle, can be applicable to almost all cases.

The concept of median line has been well known since long time to surveyors (or, to be more precise from the etymological point of view, to geodesists) who had to determine the limits of different private territory areas placed on opposite edges of lakes and rivers.

Of course, the curves of the rivers and the irregularities of the coastlines were being considered in order to get a result which could take into account possible anomalies and the lengths of the relevant coastlines.

Concerning the sea, the problem was - and actually ismore complex, first of all due to the practical impossibility of a direct measure of the distances between one coast and the other and due to the different cartographic representations of the coastlines. Nowadays, the problem can be solved using satellite positioning and remote sensing techniques; anyway, the present situation is still influenced by the heritage of the past — mostly graphical—procedures.

As far as is known to the authors, many present bilateral agreements have been stipulated making reference to an equidistance line graphically drawn on the nautical maps existing at the agreement epoch, because it was felt necessary first to agree on the principle, and then to represent visually the limit line determined according to that principle. In fact, using the positioning procedures adopted until recently, it was practically impossible to determine the position of a boat with a precision greater than several hundred meters (and the nautical maps did not have a greater precision). Navigation criteria were thus followed, rather than geodetic precision procedures; as a consequence, the graphically drawn equidistance line was to be considered sufficient and suitable to the purpose.

Basically, the Kennedy method consists in a geometrical construction using circles and segments, based on the concept of fixed-or control-point. Fig. 1 shows an

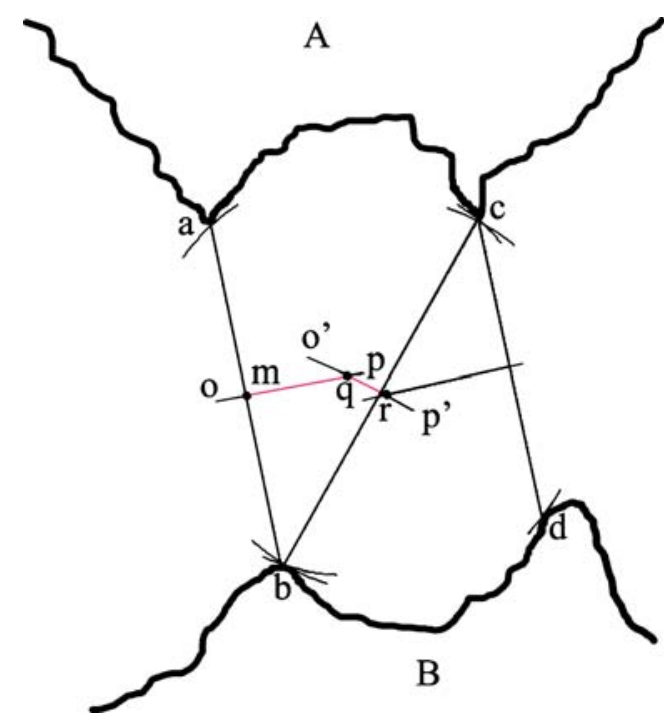

Fig. 1 Median line obtained with the classical method

example of the graphical construction for the determination of some points which can help to describe the procedure (IHO, IAG, IOC 2006):

"Taking points $\mathbf{a}$ and $\mathbf{b}$ and the segment $\mathbf{a b}$, a perpendicular bisector op of $\mathbf{a b}$ is drawn with $\mathbf{m}$ being the precise bisector point of the line $\mathbf{a b}$. Proceed towards $\mathbf{p}$ until a circle passing through $\mathbf{a}$ and $\mathbf{b}$ and with center in a point $\mathbf{q}$ of the segment op will be tangent at the coastline of one of the two States. In the figure that point is $\mathbf{c}$ in State $\mathbf{A}$. We have that $\mathbf{c}$, $\mathbf{a}$ and $\mathbf{b}$ are equidistant from $\mathbf{q}$. Now taking $\mathbf{b}$ and $\mathbf{c}$ and the segment $\mathbf{b c}$, a perpendicular bisector $\mathbf{o}^{\prime} \mathbf{p}^{\prime}$ is drawn similar to the latter case. This intersects the first baseline bisector op at q. Then proceed towards $\mathbf{p}^{\prime}$ until a circle passing through $\mathbf{b}$ and $\mathbf{c}$ and with center in a point $\mathbf{r}$ of the segment $\mathbf{o}^{\prime} \mathbf{p}^{\prime}$ will be tangent at the coastline of one of the two States. In the figure that point is $\mathbf{d}$ in the State $\mathbf{B}$. By proceeding to the right in this way the segments of the median line will be constructed until the total median line is derived."

Although in general the system fulfills the specific needs, it is lacking from the objectivity and rigor viewpoint, because the choice of the fixed points (above all the initial couple of fixed points) is operator dependent. That choice is indeed based on the consideration of the nearest points, which is often a difficult task, especially in the case that some parts of the coast (e.g., the islands' coastlines) have to be differently weighted in the computations. If this is the case, the complication of the construction increases dramatically, together with the production times.

In addition to the objectivity and rigor needs, one has to take into account also the efficiency of the process. As will be clear in the following, the computing times are in the order of some tens of minutes, while the manual procedure is in the order of tens of hours. 
Moreover, it has to be pointed out that the method works on the cartographic representation on the interested area and neglects the relevant deformations. That is completely justified from a practical viewpoint, especially considering the small scales concerned and the consequent high amount of graphic error $(0.2 / 0.4 \mathrm{~mm}$ at the map scale); however, it cannot be sufficiently justified in view of the existing computation abilities and of the possibility of adopting greater scales when particularly critical boundary situations are dealt with or when satellite positioning methods are used.

\section{The reference system and the estimation of the geodesic distance}

The above problem finds its natural environment within a geodetic reference system, always coming together with a "mathematical" surface intended to represent the earth physical surface ${ }^{2}$ as much effectively as possible. The geometric surface almost always used for that purpose within the geomatic and hydrographic applications is the biaxial (or rotation) ellipsoid, suitably sized and shaped, as a reference for the curvilinear coordinates: latitude $\varphi$ and longitude $\lambda$. A significant example of the above definitions is the modern system ETRS89 (European Terrestrial Reference System 1989) which has been adopted by almost all the European Countries: to this system, the geocentric biaxial ellipsoid GRS80 (Geodetic Reference System 1980) is associated and to this system are currently referred the absolute and relative position determinations obtained by the global positioning system (GPS) ${ }^{3}$; other examples of a geodetic reference system are the classical national geodetic systems (in Italy, the Roma'40 system) to which generally non-geocentric biaxial ellipsoids are associated (in Italy, the Hayford ellipsoid).

Thus, assuming to work with reference to, e.g., the GRS80 ellipsoid, it is useful here to briefly examine the concept of distance on the ellipsoid, intended as the length of a geodesic line on that surface.

The geodesic line on the ellipsoid

Let there be given a biaxial ellipsoid of equation

$x^{2}+y^{2}+\frac{z^{2}}{\left(1-e^{2}\right)}-a^{2}=0$

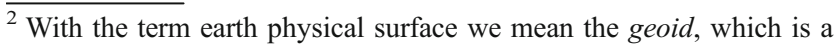
particular equipotential surface of the earth's gravity field.

${ }^{3}$ As a matter of fact, the system ETRS89 can be considered as an European "realization" of the system WGS84 (World Geodetic System 1984), which is closely connected to the GPS.
}

where $a=$ semi-major axis $e=\sqrt{\frac{a^{2}-b^{2}}{a^{2}}}=$ first eccentricity $(b=$ semi-minor axis) (for the GRS80-a=6,378,137.0 $\mathrm{m}, e^{2}=$ $6.694380023 \times 10^{-3}$ ).

A geodesic line is defined as a curve of the ellipsoidal surface such that, for each point $\mathrm{P}$ of the curve, the principal normal $\mathbf{n}$ to the curve at $\mathrm{P}$ and the normal $\mathbf{N}$ to the surface at $\mathrm{P}$ coincide:

$\mathbf{n}=\mathbf{N}$

In simpler terms, since the direction of the principal normal of a space curve at $\mathrm{P}$ is that direction along which an observer would see rectilinear the curve in a neighborhood of the point, the above condition applies that property on the ellipsoidal surface, which means that a geodesic line is a locally rectilinear curve with respect to the ellipsoidal surface. Furthermore, one can prove that a geodesic line is a curve with minimum length ${ }^{4}$ and it is just this feature which makes it geometrically suitable to represent a distance.

The length of a geodesic line can be computed very accurately-even for very long lines-using suitable numerical methods which can be easily implemented in effective software codes (see, for instance, Birardi 1988).

However, for the purposes of the present work, it can be sufficient to adopt, e.g., the simpler procedure described in (Coticchia and Surace 1978) which uses more concise formulas and provides the length of an ellipsoidal geodesic line with a precision of few centimeters upon distances up to $100 \mathrm{~km}$.

\section{The method of the equidistant point (EP)}

Consider now a single point and two point sets on a surface whatsoever, provided with a metric $d$. In general, the distance $d(\mathrm{X}, \alpha)$ between a point $\mathrm{X}$ and a point set $\alpha$ is meant as the infimum (in mathematical terms) of the distances between $\mathrm{X}$ and each of the points belonging to $\alpha$. Then we can state the following definition:

Given a family $\Omega=\left\{\alpha_{i}: i=1, \ldots, N\right\}$ of point sets $\alpha_{i}, X_{0}$ is called an equidistant point (EP) if:

1. $\exists j, k \in\{1, \ldots, N\}: d\left(X_{0}, \alpha_{j}\right)=d\left(X_{0}, \alpha_{k}\right)$;

2. $\forall i \in\{1, \ldots, N\}$ with $i \neq j$ and $i \neq k: d\left(X_{0}, \alpha_{i}\right) \geq d\left(X_{0}, \alpha_{j}\right)$.

The determination of $X_{0}$ of course depends on the definition of the particular metric which is being adopted. However the concept of EP is clearly independent, and also the case of a different weighting of certain point sets (in order to reduce or increase the computed distance depending on the circumstances) can be included whatever the choice of the metric.

\footnotetext{
${ }^{4}$ For surfaces whatsoever that is true just in a local, finite sense.
} 
In the practical applications, which will be illustrated in the following sections, the notion of geodesic distance presented in the section 'The reference system and the estimation of the geodesic distance' for the WGS84 (ETRS89) ellipsoid has been adopted.

\section{The EP determination}

The case of two coastlines

Consider two different point sets (we shall see that the case with more than two point sets can be reduced to this one) and apply the procedure to particular point sets, such as the coastlines, belonging to an ellipsoidal surface. We shall adopt the usual geographic reference system with latitude and longitude coordinates.

Consider first the case with two coastlines, which is the most frequent scenario.

It is essential to introduce a bi-dimensional mesh of points with a defined interval $\Delta \Phi$ in latitude and $\Delta \Lambda$ in longitude. ${ }^{5}$

As will result clearer in the following, the method which is being illustrated here-on which the relevant algorithm is based - consists in the examination of each of the mesh points in order to verify (or not) the equidistance condition from the two point sets representing the two coastlines, respectively.

Before proceeding further, anyway, it seems necessary to give an approximate estimation of the error which will affect the coordinates of the EP which are being determined with this procedure. That can be obtained on the basis of the following semi-rigorous considerations: assuming for a moment that the coastline points coordinates are error free, since the intervals of the mesh have a finite length, it is evident that the condition of equidistance can be verified with a maximum "mesh error" of $\pm \frac{1}{2} R \Delta \Phi$ in latitude and of $\pm \frac{1}{2} R \cos \Phi \Delta \Lambda$ in longitude, where $R$ is the radius of the local sphere at the latitude $\Phi$. Anyway, the coastline points coordinates are affected also by the position errors and, in particular, the coordinates of the points, called FP1 and FP2 (fixed, or control, points), of the two coastlines for which the condition of equidistance to EP is verified. As a consequence, we have to consider also the mean square errors (m.s.e.) $\pm \sigma_{F P}^{\varphi}$ and $\pm \sigma_{F P}^{\lambda}$ relevant to the latitude and to the longitude, respectively, of those points ${ }^{6}$. As a matter of fact, such position errors reflect on the corresponding position errors relevant to the point EP in the following way: Let $d x_{\mathrm{EP}}$ be the true error, along any direction, of the

\footnotetext{
${ }^{5}$ As will be better explained in the following, such intervals can be reduced conveniently depending on possible needs of greater graphical details.

${ }^{6}$ Here we suppose, for simplicity, that the coordinates of all points of both the coastlines are affected by the same error in latitude and by the same error in longitude.
}

position of EP: it is clear that, due to the equidistance of EP from FP1 and FP2, such error is given by

$d x_{E P}=\frac{d x_{F P 1}+d x_{F P 2}}{2}$

where $d x_{\mathrm{FP} 1}$ and $d x_{\mathrm{FP} 2}$ respectively represent the true errors of FP1 and FP2 along the same direction. From Eq. (2), according to the error propagation law, we get

$\sigma_{E P}^{2}=\frac{1}{4} \sigma_{F P 1}^{2}+\frac{1}{4} \sigma_{F P 2}^{2}=\frac{1}{2} \sigma_{F P}^{2}$

where the last equality is a consequence of the simplifying hypothesis (see the previous Note 6). Applying again the $\mathrm{m}$. s.e. propagation law, and including also the mesh errors, the following total errors can be found for the latitude and the longitude, respectively:

$\sigma_{E P}^{\varphi}= \pm \frac{1}{2} \sqrt{R^{2} \Delta \Phi^{2}+2\left(\sigma_{F P}^{\varphi}\right)^{2}}$

and

$\sigma_{E P}^{\lambda}= \pm \frac{1}{2} \sqrt{R^{2} \cos ^{2} \Phi \Delta \Lambda^{2}+2\left(\sigma_{F P}^{\lambda}\right)^{2}}$

As already noted, the above values provide just an approximate estimation of the error of the coordinates of EP.

Just for the sake of illustration, Fig. 2 shows a possible dislocation of "EP candidates" (belonging to the mesh) around the ideal equidistance line.

Taking into account the above considerations, it appears even more essential here to complete the notion of point with the uncertainty of its determination (on the surface or even in the space). As is well known, such uncertainty is covered, on the maps, by the "graphic error", ; anyway, considering a digital, vectorial representation of a surface, like the ellipsoid, in the space, the scale and graphic error notions lose much of their importance and the measured position of a point, together with its uncertainty, becomes fully significant instead.

Therefore, the main feature of this method is to consider the equidistance line and the coastlines as point sets and not as lines. It is not a restriction at all because, graphically, the coastline is always represented by a polygonal line which, of course, can be substituted by a more or less dense point set, depending on the chosen accuracy. If the purpose is just to determine the point set constituting the median line with a given accuracy, it is immediately understandable the advantage of a method which does not need to rest on geometrical/analytical considerations. As a matter of fact, methods resting on analytical formulas (Wahl 1990-Milan Horemuž et al. 1999-Murphy et al. 1999) are essentially based on the computation of the turning points (TP)

\footnotetext{
${ }^{7}$ The "graphic error" on a technical map is defined as the minimum distance at which two points can be seen by the human eye as separated. Usually it amounts to $0.2 \mathrm{~mm}$.
} 
Fig. 2 Median line obtained with the EP method

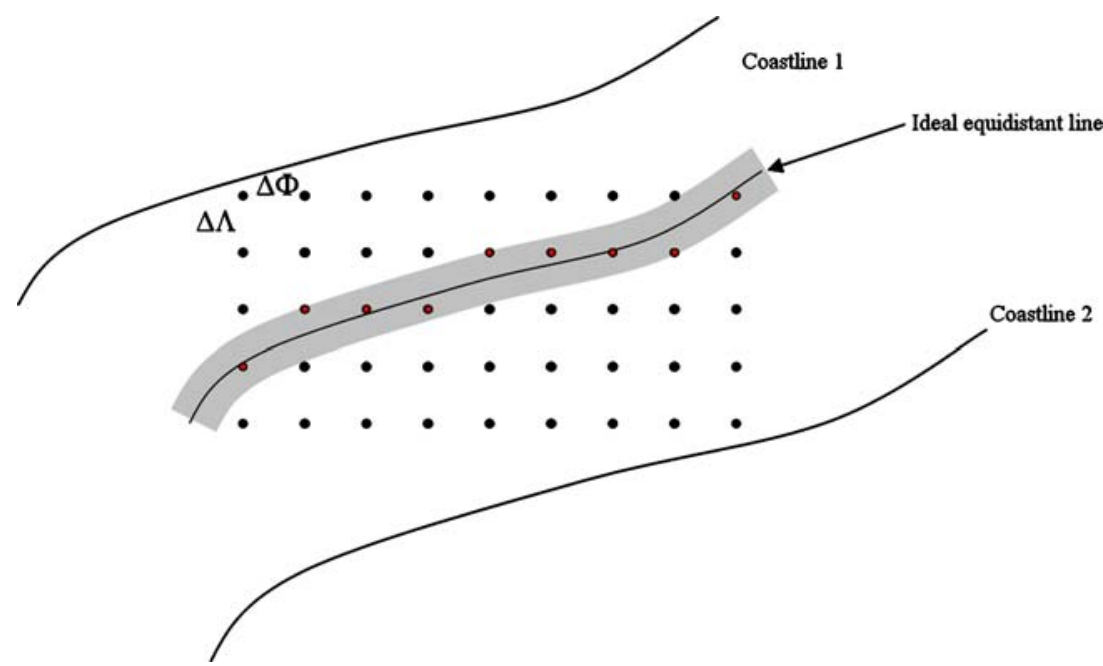

considering a number of basepoints, not more than five points each time, on the coastlines and distinguishing among the different reference surfaces with different topologies. That approach, although sensibly contributing to the solution of the problem from an analytical viewpoint, does not seem convenient, in computational terms, with respect to the one based on numerical methods; it should also be taken into account the complexity of the coastlines and the considerable amount of points (of the order of thousands) which are necessary to describe them. Instead, the method which is being discussed here does not start from the basepoints in order to search for the TP: it simply tests all the surface points in the mesh to find out which of them are good candidates as TP and, as already noted, does not depend on the choice of the topology. Therefore, this method is basically operational and, implemented in a simple algorithm (Fig. 3), is well suited to be translated into a software.

Of course, it is also conceivable to develop optimized procedures for the search of the EPs. It is possible, e.g., to start from a mesh with large-sized intervals and then to decrease the size more and more until converging to the EPs with a minimum mesh error (obviously it will not be possible to decrease the amount of the position errors of the FPs).

The development of the test software has been carried out with the platform $\mathrm{C}++$.NET using a convergence criterion for the progressive refinement of the mesh interval size.

Using a normally equipped PC (AMD Athlon 64-bit $2 \mathrm{GHz}-2 \mathrm{~GB}$ RAM) and working on two coastlines composed by 4,991 and 8,000, respectively, on an area of amplitude $2^{\circ} 30^{\prime}$, a mesh interval of $0.1^{\circ}$ both in latitude and in longitude and a test precision of $0.00001^{\circ}$, a line composed by 4,183 points was obtained (having established that the indeterminacy should not be greater than $1 \mathrm{~m}$ along the meridian and along the parallel). The total computation time was around $10 \mathrm{~min}$. The coastlines in the following examples are real coastlines but their locations may be fictitious in order to obtain the different cases of study. The

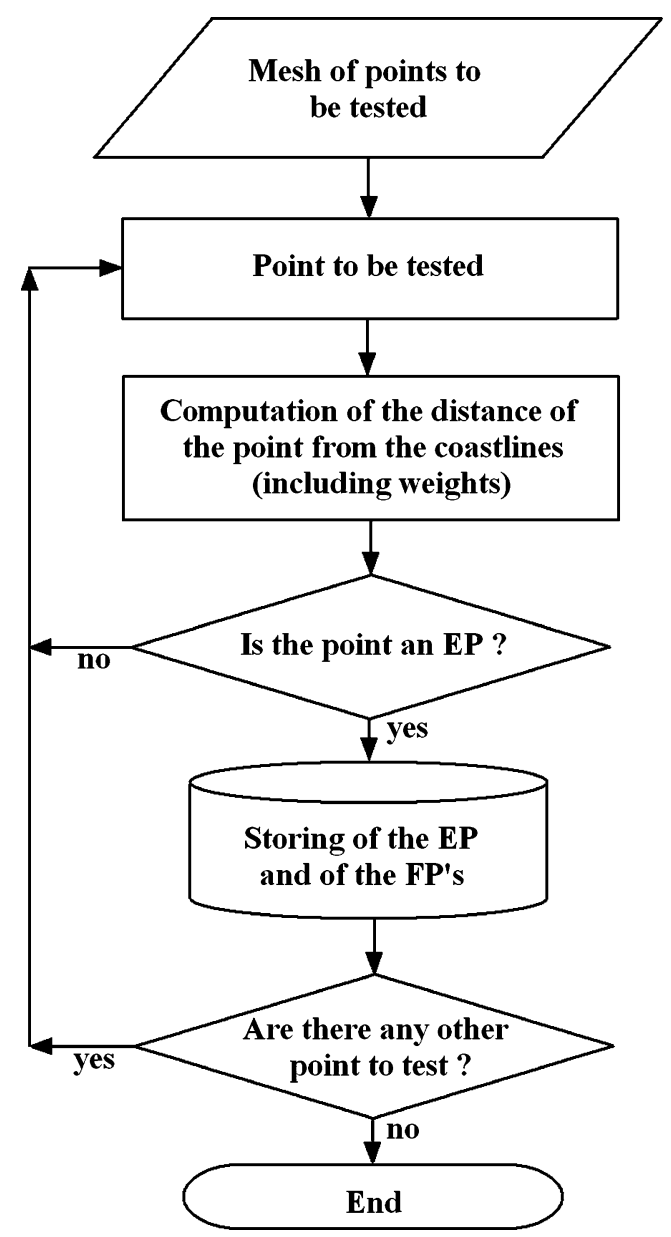

Fig. 3 The algorithm of the EP method 


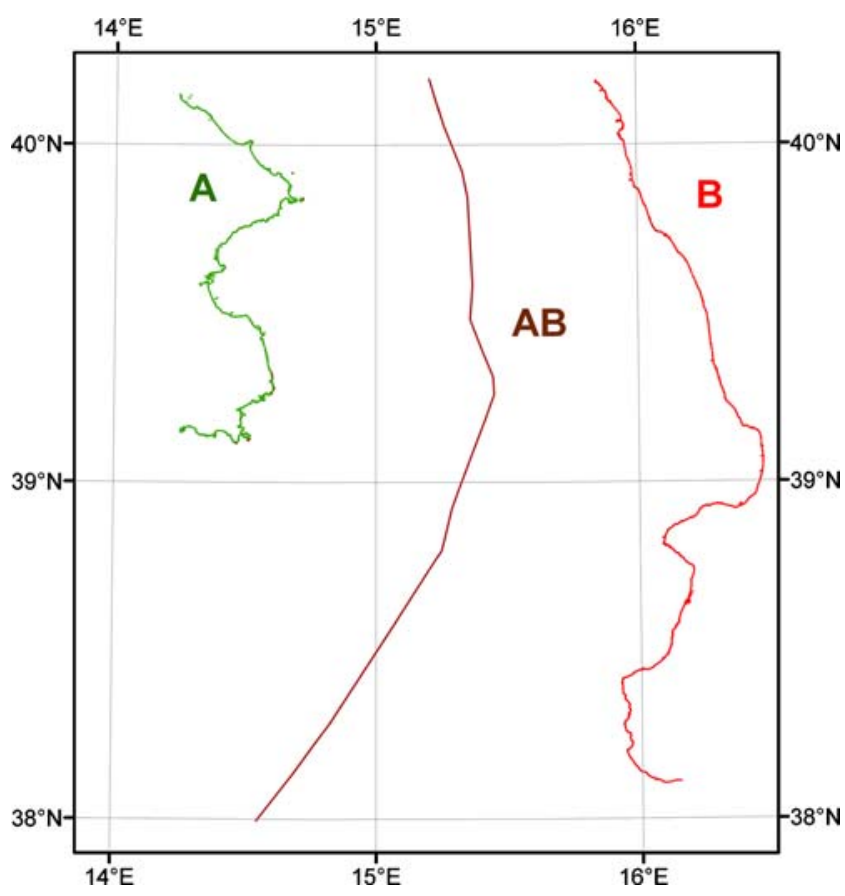

Fig. 4 The case of two coastlines

graphical output for the case of the two coastlines is reported in Fig. 4.

In Fig. 5, the case of two adjacent coastlines is represented. On the coastlines, the control points are indicated in red, i.e., those couples of points on the coastlines which are located at a minimum distance from an EP on the median line and which are computed together with the EPs.

Fig. 5 The case of two adjacent coastlines

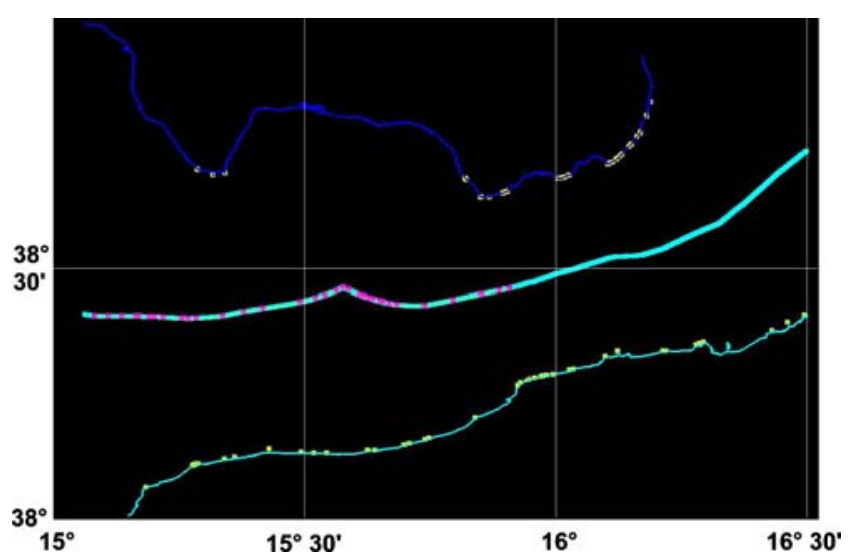

Fig. 6 The comparison between the EP (cyan) and the classical method (purple)

Furthermore, a comparison has been performed with the results obtained through the graphical procedure based on the conventional method cited in 'The classical method'. For practical reasons, due to the long time required to obtain an equidistant line with the classical method, we took a previous work, so the coastlines of Fig. 6 do not resemble any of the previous ones.

The comparison results are presented in Figs. 6 and 7. In both figures, the purple squares are the points obtained with the graphical procedure, while the cyan ones are those obtained with the present method. The dimensions of the rectangle considered in Fig. 6 are $1^{\circ}$ in latitude and $1^{\circ} 31^{\prime}$ in longitude.

The construction lines have been omitted in order to make the graphics more understandable. Figure 7 reports a detail which evidences the substantial coincidence of the

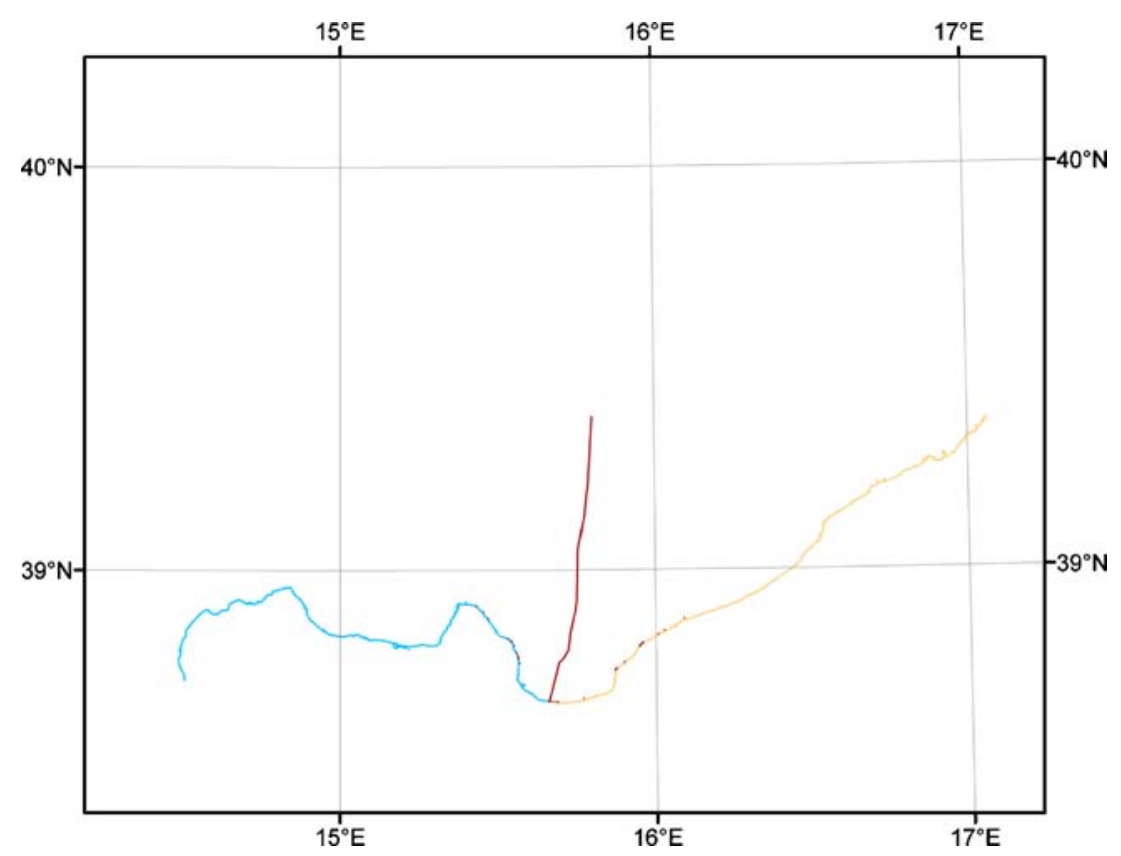




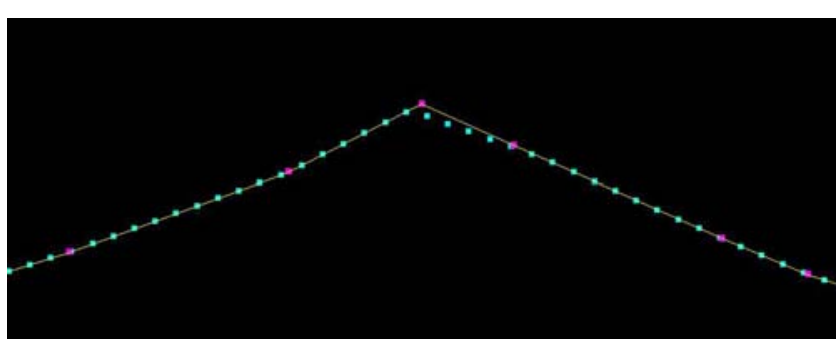

Fig. 7 Zooming a turning point. The mean distance between cyan points is $250 \mathrm{~m}$

two methods, with the exception of the cusp zone where the graphical method shows a difference in the "critical" cusp point. Such point is the well-known turning point (TP).

The present method and the turning points (TP)

In the method based on the geometrical construction, the median line turns out to be a polygonal line connecting the TPs. As already noted, they are the points at which the median line changes its direction depending on the profiles of the near parts of the coastlines. With the present method, instead, substantially based on numerical computations, the median line is composed by a point set whose density can be freely chosen and which does not include, in general, the TP. Then, similarly to the results obtained with the graphical method, one could include a control in the algorithm for identifying a TP among the EPs depending on the variation of one of the two fixed points, in order to get only the TPs as output. As an

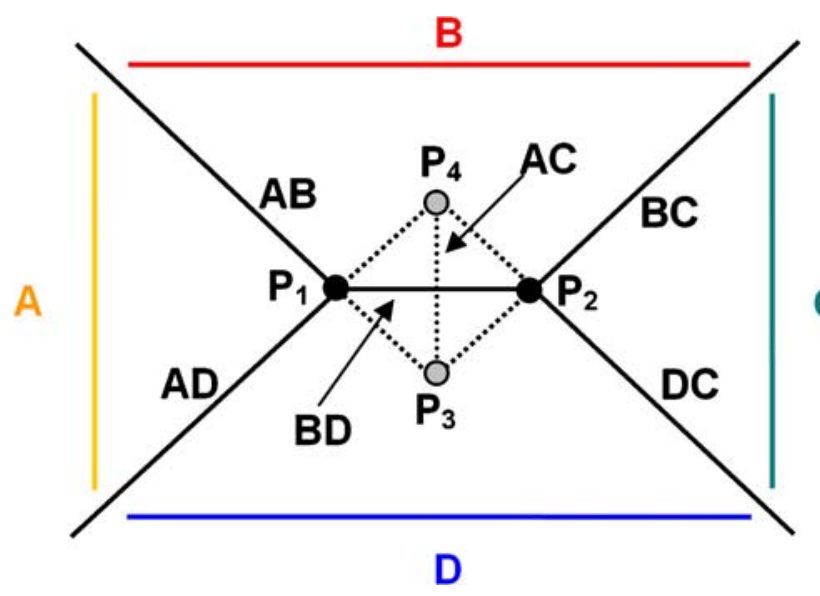

Fig. 9 The simplified case of four coastlines

alternative, the formulas by Sjöberg et al. (Sjöberg 2001; Carrera 1987; Horemuž et al. 1999) for the computation of the TPs could be used, where the three necessary points are the two fixed points plus the point which is varied on one of the two coastlines.

In any case, it should be observed that the present method has been conceived mainly to fulfill an operational need, which is to obtain a satisfactory result within the given precision.

On the other hand, also the exploitation of exact geometric formulas for the electronic computation of the TPs should be done through numerical methods in order to apply to the resulting median line all the previous

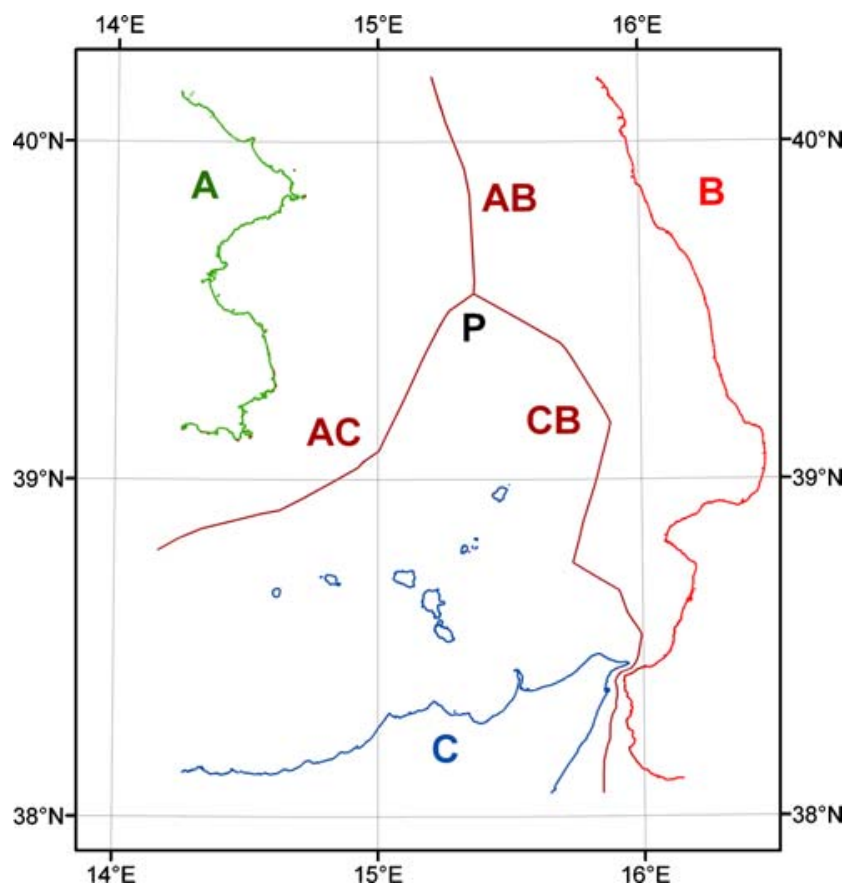

Fig. 8 The case of three coastlines

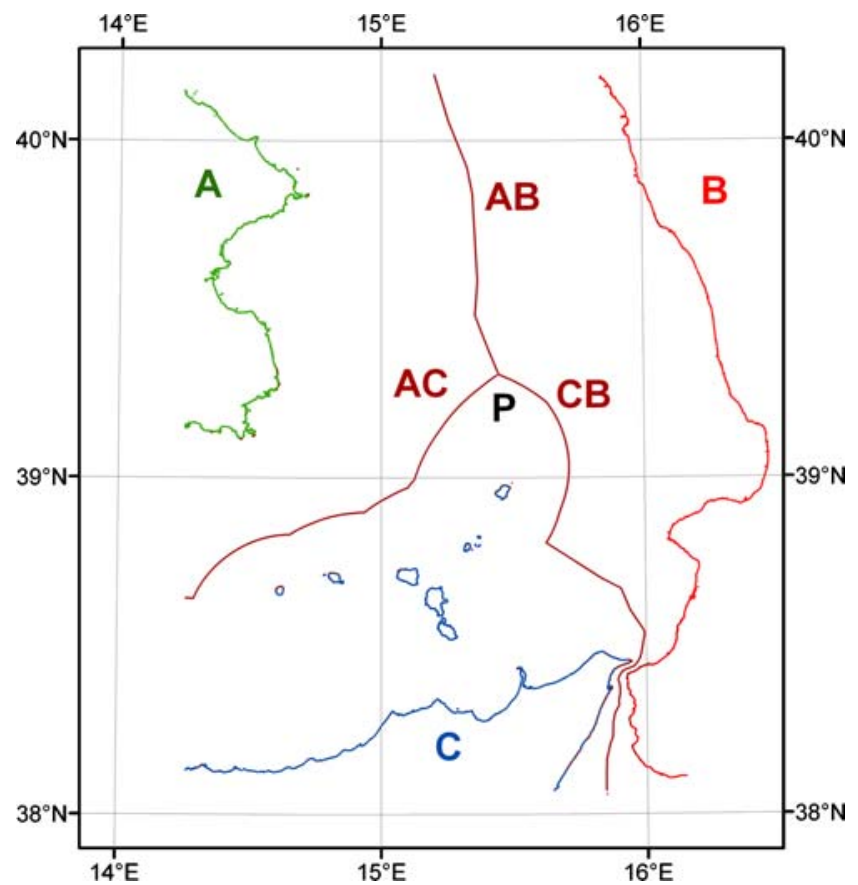

Fig. 10 The case of weighted coastline (blue) 


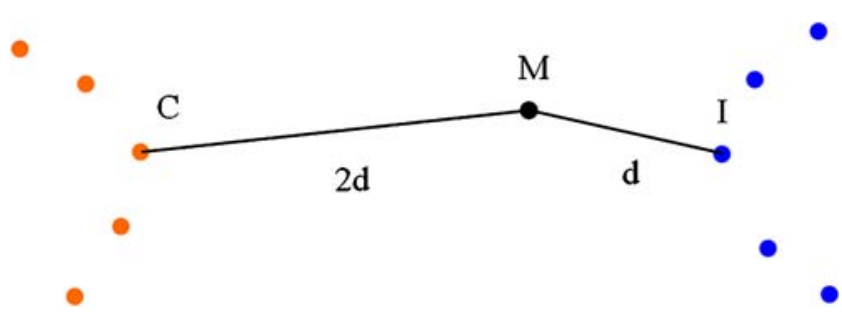

Fig. 11 The explanation of the weighting method

considerations on the precision. As a matter of fact, the coastlines may have whatever shapes and one often finds many - and very close each other-TPs. That mostly happens if the coastlines are sufficiently smooth and close each other, or if they have an evident curvilinear profile (see Fig. 8, near to the islands), so that in such cases the median line results in a curve rather than a polygonal.

Moreover, this method uses a more direct approach with no need to apply formulas other than those for the computation of the geodesic distance between two points.

The case with more than two coastlines

In case we have a situation with three coastlines, a simple remark is in order: if two median lines intersect in a (equidistant) point $\mathrm{P}$, then the third median line passes through $\mathrm{P}$ too. In fact, call A, B, and C the point sets in this situation and suppose that the intersecting lines are the two median lines between $\mathrm{A}$ and $\mathrm{B}$ and between $\mathrm{B}$ and $\mathrm{C}$. Indicating with $\mathrm{La}, \mathrm{Lb}$, and $\mathrm{Lc}$, the distances between the intersection point $\mathrm{P}$ and the point sets $\mathrm{A}, \mathrm{B}$, and $\mathrm{C}$, respectively, we have $\mathrm{La}=\mathrm{Lb}$ and $\mathrm{Lb}=\mathrm{Lc}$, due to the definition of $\mathrm{P}$ as an equidistant point between $\mathrm{A}$ and $\mathrm{B}$ and between $\mathrm{B}$ and $\mathrm{C}$, from which it follows $\mathrm{La}=\mathrm{Lc}$. Thus, $\mathrm{P}$ is also equidistant between $\mathrm{A}$ and $\mathrm{C}(\mathrm{Lp}=\mathrm{La}=\mathrm{Lb}=\mathrm{Lc}$, where $\mathrm{Lp}$ represents the equal distance of $\mathrm{P}$ from each of the three coastlines). This elementary result of course is true independently from the adopted topology. This fact implies that the case with three coastlines can always be treated as that with two coastlines, considering all the possible combinations of them. Then all the median lines will intersect in a point $\mathrm{P}$ and the residual part of a median line between, e.g., A and B "going beyond" that point can be cut off, because the distance of those points from the third coastline $\mathrm{C}$ is lesser than the distance ( $\mathrm{Lp}$ ) of point $\mathrm{P}$ (according to the second condition of the algorithm criterion; see 'The method of the equidistant point (EP)'). Figure 8 presents an example illustrating the above situation with $\mathrm{P}$ representing the intersection of the three median lines.

If we have more than three coastlines, the complication of the problem's geometry increases and we cannot say anymore that the median lines obtained from the fourth coastline on and the others intersect into the same point as before. Figure 9 shows a simplified example of this situation with four coastlines from which it follows that we can have intersections made at most by three median lines at the points $\mathrm{P}_{1}$ and $\mathrm{P}_{2}$ (and where the points $\mathrm{P}_{3}$ and $\mathrm{P}_{4}$ also represent intersections of median lines which, nevertheless, have to be discarded together with the dotted lines in the figure because they do not fulfill the second condition of 'The method of the equidistant point (EP)').
Fig. 12 The computation of the territorial line

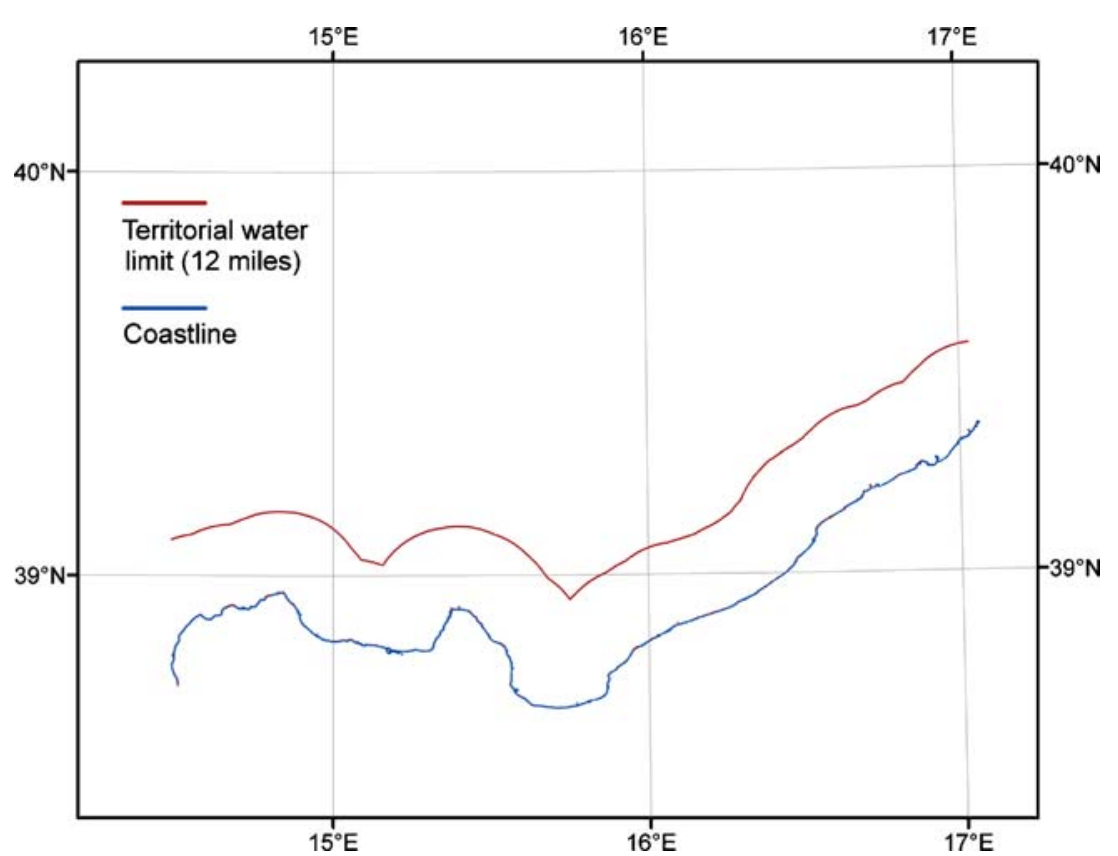


We will not proceed to consider cases with more than four coastlines due to the obviously increasing complication of the relevant geometry and also taking into account that a situation with four coastlines is already a very rare case. Nevertheless, the above situations have been mentioned here just to point out the effectiveness of the present method which, as already observed, can give up considering the specific geometry of the particular case.

\section{The case of the weighted distance}

It often happens that some parts of a coastline, or in most cases the islands, must be considered with a minor weight with respect to the rest of the coastline. In Fig. 10, a case is shown in which the islands of coast $\mathrm{C}$ have been given a weight such that the median line passes at a distance from them which is one third of its distance from coastline A, instead of passing at the same distance as in Fig. 8; note that also in this case we still have the intersection of the three median lines at a point $\mathrm{P}$.

In general, if $\mathrm{C}$ and I represent two coastlines and $\mathrm{M}$ is the mesh point to be tested, the "weighted" result is obtained simply multiplying by a given factor $n$ the value $d$ of the distance MI between the point M and the coastline I and then submitting that scaled distance to the "EP test". Figure 11 shows the situation with $n=2$. In general, the median line will pass at a fraction $1 /(1+n)$ of the distance MI + MC from I, where $n$ is the weight of the coastline I (Fig. 11).

In the determination of the median lines are also used the baselines, which usually "rectify" some parts of the coastlines or "close" bays or gulfs. In order to take into account the baselines in the algorithm, one can replace them with a set of points suitably spaced one from another depending on the precision needs.

\section{The determination of the territorial line}

The present method can also be used to determine a line located at a fixed distance from the coastline. In this case, of course it does not exist a point set representing the other coastline, so one can simply choose as EPs all those points whose distance from the coastline is the chosen distance.

With this method is also possible to determine the socalled waterlines (Christensen 2002) defined as "lines representing water, drawn parallel with the edge of a water feature, which decrease in proximity and strength away from this edge" (Neumann 1997). The most natural application is the determination of the territorial limits (Fig. 12).

\section{Conclusions}

Basically, the present method differs from the previous ones due to the starting data which in this case are the sea points which are tested. The other methods, presented in the references, always start with the points on the coastlines and then provides the TPs or the EPs (Carrera 1987).

Another peculiar feature of the method is its conceptual simplicity and its independence from the environment topology. From a theoretical point of view, it would be also possible to extend the method to the space, simply considering surfaces instead of lines (at present, however, there are no applications).

To summarize, the present method fulfills the followings requirements:

1. Objectivity. The definitions and the procedure adopted are unambiguous (and used in an algorithm).

2. Precision. The error can be defined and evaluated.

3. Effectiveness. The computation times are much less than those typical of a work carried out with CAD softwares.

4. Flexibility. The method can be applied in every circumstances and scenarios of coastlines, including islands. Moreover, it provides solutions in all the situations concerning the computation of distances from the coastlines, including the cases of the territorial limits and the waterlines.

\section{References}

Birardi G. (1988) Corso di Geodesia, Topografia e Fotogrammetria, Parte II (Geodesia Teorica), Collezione dei testi Tecnici dell'Istituto Geografico Militare (Firenze), p. 221-231

Carrera G. (1987) A method for the delimitation of an equidistant boundary between coastal states on the surface of a geodetic ellipsoid. International Hydrographic Review, Monaco, 44, 1. pp. $147-159$

Christensen Albert H. J. (2002) A fully automated sea boundary delineator. FIG XXII International Congress. Washington, D.C., USA, April 19-26, 2002

Coticchia A., Surace L. (1978) Risoluzione di problemi geodetici con le minicalcolatrici elettroniche programmabili, Boll. Geodesia e S.A., n. 1/1978, pp. 111-113

Francalanci G.P., Spanio F. (2000) La convenzione delle UU.NN. sul diritto del mare-aspetti tecnici I.I. 1131, II Ed., pp. 79-88

Horemuž M., Sjöberg L. E. and Fan H. (1999) Accuracy of computed points on a median line, factors to be considered. Proceedings of the International Conference on Technical Aspects of Maritime Boundary Delineation and Delimitation, Session 2, Paper 5 pp. 133-143

IHO (International Hydrographic Organization), IAG (International Association of Geodesy), IOC (Intergovernmental Oceanographic Commission) (2006) A manual on technical aspects of the United Nations Convention on the Law of the Sea-1982. International Hydrographic Bureau, Monaco, Special publication No. 51, 4th Edition. Chapter 6 par. 6.2, par.6.2.1 
Murphy B., Collier P., Mitchell D. and Hirst B. (1999) Maritime zone boundary generation from straight baselines defined as geodesics. Proceedings of the International Conference on Technical Aspects of Maritime Boundary Delineation and Delimitation, Session 2, Paper 4, pp 120-132

Neumann, J. (1997). Dictionary of cartography in 25 languages. K.G. Saur. 22nd Edition, 586 pages
Sjöberg Lars E. (2001) The three-point problem of the median line turning point: solutions for the sphere and ellipsoid. Royal Institute of Technology Division of Geodesy SE-100 44 Stockholm-2001 ABLOS Conference

Wahl Jerry L. (1990) A computer aided design approach to median line computations. ACSM Spring 1990 Conference, Denver (CO) 